\title{
CURRENT LITERATURE
}

\section{STATUS EPILEPTICUS IN ACUTE SETTINGS}

\section{Status Epilepticus Arising De Novo in Hospital- ized Patients: An Analysis of 41 Patients}

\author{
Delanty N, French JA, Labar DR, Pedley TA, Rowan AJ
}

Seizure 2001; 10: 116-119

Most of the information on predisposing factors and mortality in status epilepticus (SE) arises from data obtained from patients presenting to the casualty department. However, another population which is frequently seen by consultative neurologists are medically ill patients who develop SE while in hospital. These patients are often notoriously difficult to treat once SE arises. We sought to characterize patients at risk for SE arising when they are hospitalized for other reasons. By doing this, risk factors for developing SE and prognostic indicators might be determined. We retrospectively reviewed records from three urban hospitals in the United States to identify hospitalized patients developing SE over a 1 year period. SE was defined as a clinical seizure lasting 30 minutes or longer, or repeated seizures without recovery. Patients who were admitted in SE or for an epilepsy-related problem, or who were less than 1 year old were excluded from the study. Forty-one patients with in-hospital SE were identified. There were 28 males and 13 females with an age range from 1 to 91 years (mean: 60 years, median: 65 years). The mean interval from hospital admission to the onset of status epilepticus was 26 days. Nineteen (46\%) patients had a prior history of either epilepsy or symptomatic seizures, and of these, 10 were inadequately treated as judged by serum anticonvulsant levels at the time SE developed. Focal brain abnormality was present in $26(63 \%)$ patients, the most common of which was stroke (17 patients). Major metabolic derangements including hypoxia, electrolyte imbalance, hepatic encephalopathy, and sepsis were present in $23(56 \%)$ patients. Eleven $(27 \%)$ patients were being treated with theophylline preparations at the time SE developed. Mortality in this group of patients with in-hospital SE was 61\% (25 deaths), with about one-third dying while in status, and two-thirds dying subsequently in hospital. In this retrospective study, there was no clear relationship between mortality and the duration of SE in this group of patients. In-hospital development of SE is usually related to underlying focal brain abnormality, especially stroke, in combination with systemic metabolic derangement. Prognosis is poor, and appears to be more related to underlying conditions rather than to status duration. More accurate prospective studies are warranted.

\section{Status Epilepticus After Stroke}

Velioglu SK, Ozmenoglu M, Boz C, Alioglu Z

Stroke 2001;32:1169-1172

BACKGROUND AND PURPOSE: Objective of our study was to determine the risk and predictive factors of status epilepticus (SE) after stroke.

METHODS: From 1988 to 2000, 1174 patients were admitted to the Department of Neurology at the Karadeniz Technical University Farabi Hospital with first-time strokes. Of these, 180 patients had poststroke first-time seizures (PFSs). We followed these 180 PFS patients for an average of 3.7 years or until death to determine the occurrence rate of SE. By comparing these data with those of PFS patients without SE, we investigated whether there were significant differences.

RESULTS: A total of 17 of the 180 PFS patients (9\%) had SE. There was no relationship between the occurrence of $S E$ and stroke risk factors, stroke type (ischemic or hemorrhagic stroke), stroke topography and cause, cortical involvement, size of lesion, seizure type, or electroencephalographic findings. SE occurred more frequently among patients with a higher disability rating (Rankin scale $>3$; odds ratio, 4.36). Recurrent SE was identified in 5 of 17 patients with SE. In all 5 of these patients, the first episode of SE occurred within the first 7 days after stroke (early-onset SE). Statistical analysis demonstrated that early-onset $\mathrm{SE}$ was associated with a higher risk for $\mathrm{SE}$ recurrence $(P=0.003)$ and a higher mortality rate $(P=0.04)$. 
CONCLUSIONS: SE was not associated with a higher mortality rate but with higher functional disability. We also found that early-onset SE (within the first 7 days after stroke) was associated with a higher risk for SE recurrence and a higher mortality rate than late-onset SE (after 7 days after stroke).

\section{COMMENTARY}

Stroke is a leading cause of acquired epilepsy and is the most common cause of status epilepticus (SE) in adults. Two recent articles examined the occurrence of SE in the setting of stroke and other acute conditions.

Delanty et al. reviewed $41 \mathrm{SE}$ cases that occurred de novo in hospital patients, excluding those who were admitted for SE or epilepsy-related problems. On average, SE occurred after 26 days of hospitalization and had a $61 \%$ mortality, which is far higher than has been found in large studies that included patients admitted for SE (1-3). This high mortality was not associated with SE duration and was felt to be due to serious comorbid conditions. Twenty percent had a history of epilepsy, and $27 \%$ experienced a single seizure while in the hospital prior to onset of SE. Of 19 patients with one or more prior seizures, 6 had been treated with antiepileptic drugs, but only 2 had therapeutic antiepileptic drug levels, suggesting that some cases of SE were potentially preventable.

This study describes a distinct subgroup of SE patients with unique risk factors and high mortality. The majority of patients in this study had a focal brain lesion, most frequently recent or remote stroke (17 patients). A recent study (reviewed in this issue of Epilepsy Currents) found that cultured hippocampal neurons exposed to glutamate (which is released in the setting of brain ischemia and anoxia) developed spontaneous recurrent epileptiform discharges (4). This model suggests that the high mortality seen in SE patients with stroke may be caused by synergistic excitotoxic effects of ischemic and epileptic injuries.
Velioglu et al., in a retrospective study, found that 180 of 1174 patients with first-time strokes had new onset of seizures following stroke. Nine percent of these seizure patients $(1.4 \%$ of the first-time stroke population) experienced SE. The authors were careful to include first-time strokes only, thereby ensuring that seizures or SE were due to an acute, rather than remote, ischemic event. The authors limited their comparison groups to 17 patients with poststroke SE and 163 patients with poststroke seizures. The only significant risk factor distinguishing these two groups was the disability rating, which was higher for SE patients. The relevance of this finding is unclear, as stroke size, type, territory, and risk factors did not differentiate the SE from the seizure groups.

The article confirms that an acute cerebrovascular event may cause self-limited as well as prolonged epileptic events, but it offers little insight as to why some are prolonged and others are limited in duration. No treatment data are presented, and thus, it remains unclear whether shorter seizures were treated more quickly or effectively or whether seizure durations in SE patients were prolonged because of a lack of treatment or refractoriness to treatment. A comparison of these groups with new stroke patients who did not experience seizures might yield more clinically useful data that would aid in determining which patients are at high risk for developing SE.

\section{References}

1. Towne AR, Pellock JM, Ko D, DeLorenzo RJ. Determinants of mortality in status epilepticus. Epilepsia 1994;35:27-34.

2. Logroscino G, Hesdorffer DC, Cascino G, Annegers JF, Hauser WA. Short-term mortality after first episode of status epilepticus. Epilepsia 1997;38:1344-1349.

3. Lowenstein DH, Allredge BK. Status epilepticus at an urban public hospital in the 1980s. Neurology 1993;43:483-488.

4. Sun DA, Sombati S, DeLorenzo RJ. Glutamate injury-induced epileptogenesis in hippocampal neurons: an in vitro model of stroke induced “epilepsy.” Stroke 2001;32:2344-2350.

by Elizabeth Waterhouse, M.D. 